\title{
Experimental and numerical investigation on the components of a pantograph slider suspension
}

\author{
Paweł Zdziebko ${ }^{1, *}$, Adam Martowicz ${ }^{1}$, and Tadeusz $\mathrm{Uhl}^{1}$ \\ ${ }^{1}$ AGH University of Science and Technology, Faculty of Mechanical Engineering and Robotics, Department of Robotics and \\ Mechatronics, Al. A. Mickiewicza 30, 30-059 Krakow, Poland
}

\begin{abstract}
Mechanical properties of the components of a pantograph's slider suspension system have large influence on the quality of pantograph-catenary interaction. In the paper the authors present the results of their experimental research on the springs that are used in the pantograph being currently in operation in Europe. Static and dynamic tests were performed, which were aimed at determining the stiffness and damping coefficients respectively. Subsequently, the procedure for automated numerical model building for the pantograph's springs was prepared employing the Python programming language and the MSC Marc solver. When a spring model is build (accordingly to the geometric properties of the springs used in tests), the elaborated algorithm iteratively tunes the material properties and computes static and dynamic load-cases, making direct reference to the experimental procedure. After completing several iterations the numerical model is finally validated, and proper material properties, as the Young modulus and the coefficients of Rayleigh damping model, are found. Then, the obtained model can be used to determine the damping and stiffness coefficients for springs characterizing various diameters, wire diameters, numbers of turns, etc. The presented modelling tool is useful for determining the pantograph sliders suspension characteristics.
\end{abstract}

\section{Introduction}

A railway pantograph takes part in the power supply process to a train. It is desired to maintain mechanical contact between a pantograph slider and a catenary wire regardless of the speed of train travel. An exemplary configuration of the pantograph - catenary system is presented in Figure 1.

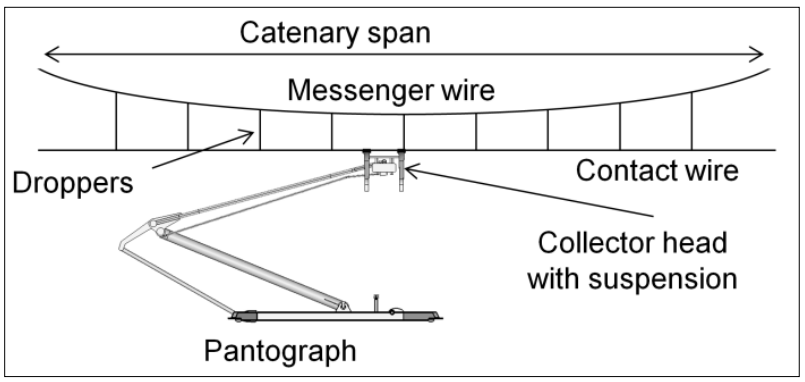

Fig. 1. An exemplary pantograph - catenary configuration.

The contact is especially disturbed in high speed runs, like it was studied in the authors previous works [1-3] and by other authors [4-7]. The most of recent research concerns pantograph refinements for improving the interaction with catenary. Many works have been made in recent years on the active solution for pantograph control to reduce fluctuations of the contact force [8-10]. The pantograph interaction with catenary can also be tuned in a passive way by reducing the mass of the collector head, and also by tuning the stiffness and damping properties of the collector head suspension system. This approach was investigated among the others in [11-13]. In these articles the authors analyze various combinations of the stiffness and damping coefficients of the simplified lumped parameters model of a pantograph. For each particular combination, analysis of interaction with a catenary was done employing an adequate simulation approach. Than the best combinations were indicated concerning improving the pantograph - catenary interaction quality. The stiffness and damping coefficients of the lumped model have been analyzed within the accepted range, but no spring has been identified to fulfil the proposed parameters (concerning geometrical shape: number of turns, spring diameter, wire diameter etc.). In this paper the authors focus on development of a numerical tool, which could be utilized to assess the stiffness $(\mathrm{K})$ and damping ratio $(\zeta)$ for any spring with various geometrical and material properties.

Initial choice of the approximate spring coefficient can be made employing the following formula:

$$
k=\frac{G d^{4}}{8 n D^{3}}
$$

where:

$k$-stiffness coefficient $[\mathrm{N} / \mathrm{mm}]$,

${ }^{*}$ Corresponding author: zdziebko@agh.edu.pl 
$G$ - modulus of rigidity of the spring material $[\mathrm{MPa}]$,

$d$-wire diameter [mm],

$D$-coil diameter [mm],

$n$ - number of active coils [-].

However setting the damping ratio of a spring (which comes from the material damping) is more difficult. Therefore, the authors decided to employ the Finite Element (FE) Method to assess the stiffness and damping ratio of the springs with different geometrical parameters. When stiffness and damping properties are assessed for a particular type of the spring, these parameters can be used as an input data for investigation of a pantograph - catenary dynamic interaction, e.g. employing the model presented previously by the authors in [1], and then the best spring shape can be found. To gain confidence about numerical results, the numerical model has to be validated. Therefore the authors performed an identification of the stiffness and damping coefficients for the springs used currently in the 160ECT pantograph produced by the Polish company EC Engineering. The results of numerical model validation are presented in this article.

The paper is divided as follows: section 2 describes experimental results of the research on the pantograph springs, section 3 describes validation algorithm proposed by the authors, section 4 presents validation results and finally section 5 summarizes the paper.

\section{Experimental research on the pantograph springs}

In this research, the springs used in the collector head suspension system of the 160ECT pantograph were investigated. In this pantograph, four springs support the collector head. Static and dynamical tests were performed to assess the stiffness and damping coefficients respectively.

\subsection{Static tests}

The static testing involves compressing the collector head spring. The tests were performed using the Instron 8872 hydraulic testing machine equipped with the force sensor, adapted to the measuring range of $\pm 200 \mathrm{~N}$ (which sufficiently covers the spring working conditions).

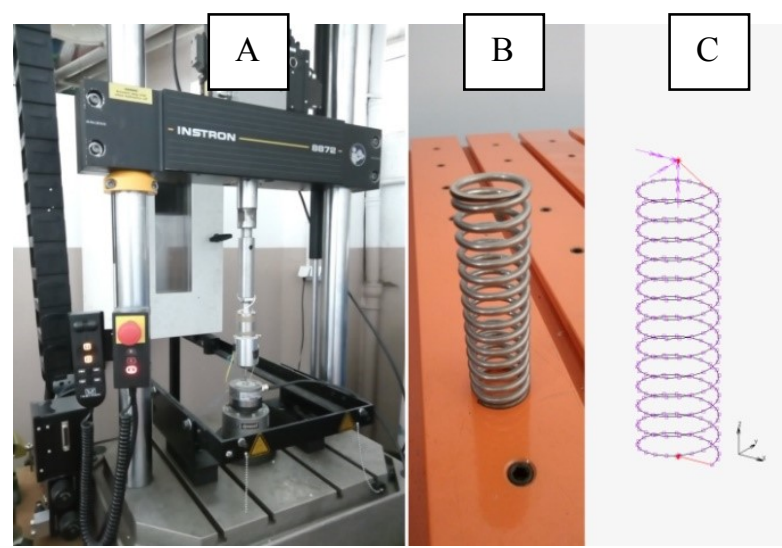

Fig. 2. Static tests: A: test stand, B: exemplary tested springs, $\mathrm{C}$ : FE model of the spring generated by the developed computational setup.
Figure 2 presents the test stand for static testing (A), and one of the tested springs of the pantograph collector head suspension system (B). In total, four springs were tested. During the static test, spring compression was registered as well as the corresponding spring force. High repeatability was noted for the tested springs. For each of them the mean stiffness coefficient was calculated based on the recorded data. The results are presented in Figure3.

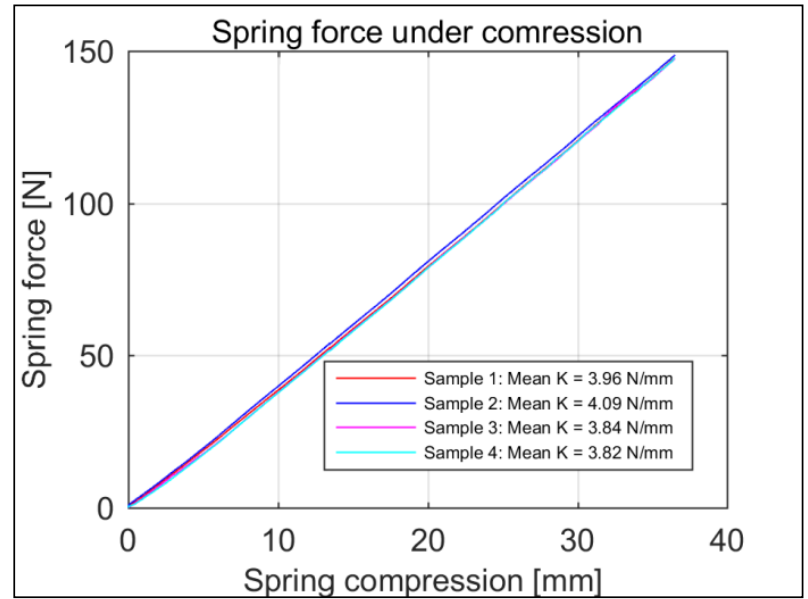

Fig. 3. Results of static tests.

\subsection{Dynamic tests}

Dynamic tests were performed on the test stand composed of the following components: tested spring with fixed support, weight rigidly attached to free end of tested spring, accelerometer mounted on the free end of the spring, signal recorder and PC computer for data preview. The test stand for dynamic tests is presented in Figure 4.

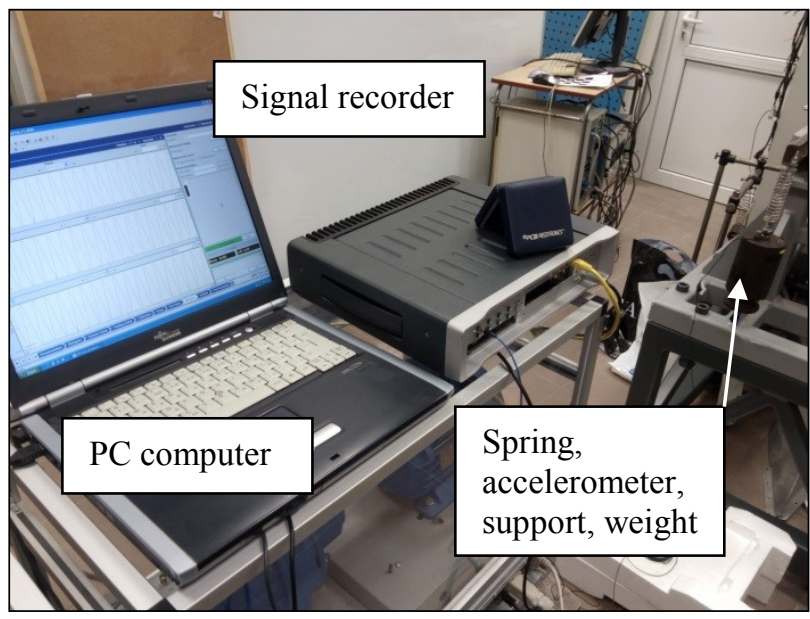

Fig. 4. Test stand for dynamic tests.

At the beginning of the test, the system was forced to lose equilibrium by impulse force acting on the weight (which represents approximately $1 / 4$ of the pantograph collector head mass). Then the accelerometer registered the oscillations of the system and data was recorded on the measuring computer. Similarly to the static tests, dynamic tests were repeated individually for all of four springs. An exemplary course of the registered 
oscillations is presented in Figure 5 (meaningless part of the signal was cut out).

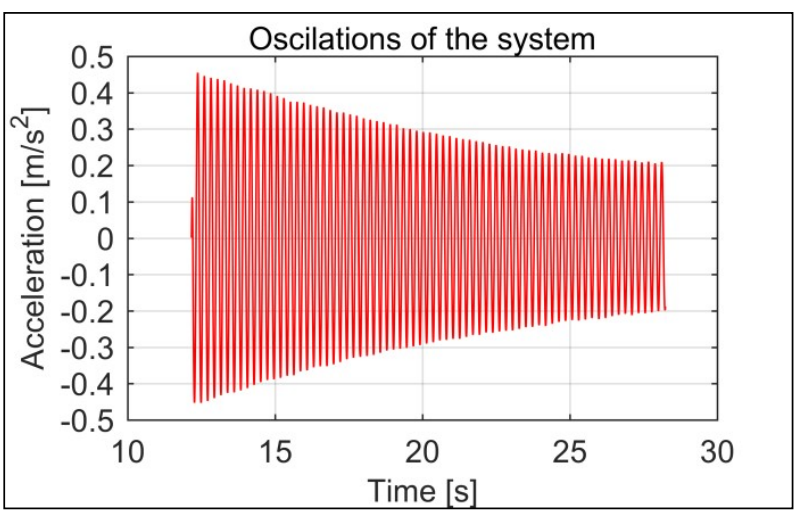

Fig. 5. Exemplary results of dynamic test.

Subsequently, employing the logarithmic decrement ( $\delta$ ) equation (see Equation 2), the damping ratio $(\zeta)$ was calculated according to the Equation 3.

$$
\delta=\frac{1}{n-1}\left(\ln \frac{x_{1}}{x_{n}}\right)
$$

where:

$\delta$ - logarithmic decrement, [-],

$\mathrm{n}-$ crest number, [-],

$\mathrm{x}_{\mathrm{n}}$-amplitude at the $\mathrm{n}^{\text {th }}$ crest number, $\left[\mathrm{m} / \mathrm{s}^{2}\right]$,

$$
\zeta=\frac{\delta}{\sqrt{4 \pi^{2}+\delta^{2}}}
$$

where:

$\zeta$-damping ratio, [-].

The logarithmic decrement was calculated for every $10^{\text {th }}$ peak of the signal, and then mean value was derived. Concluding the experimental part of this investigation, performed tests allowed for stiffness and damping coefficients designation. The results for analysed springs are presented in Table 1 . The registered results are repeatable, therefore calculated mean values of $\mathrm{K}$ and $\zeta$ are used in numerical model validation: $3.93 \mathrm{~N} / \mathrm{mm}$ and 0.00175 for stiffness and damping ratios, respectively.

Table 1. Experimental results of the spring rate and damping ratio.

\begin{tabular}{|l|l|l|}
\hline & $\mathrm{K}[\mathrm{N} / \mathrm{mm}]$ & $\zeta$ \\
\hline Spring 1 & 3.96 & 0.0018 \\
\hline Spring 2 & 4.09 & 0.0018 \\
\hline Spring 3 & 3.84 & 0.0017 \\
\hline Spring 4 & 3.82 & 0.0017 \\
\hline MEAN & 3.9275 & 0.00175 \\
\hline
\end{tabular}

\section{Algorithm for numerical model validation}

In this article the authors present a numerical tool for validation of a three dimensional FE model of a spring. The goal of this work is to build and tune the numerical model of the collector head suspension springs. The authors decided to utilize the FEM for preparing the three dimensional model of the spring with the weight connected to its one end and with fixed other end (the same boundary conditions as in the experimental tests). The algorithm includes automatic model generation and validation of static and dynamic properties of the spring. The stiffness coefficient and damping ratio. presented in the previous section, are necessary to be measured first, because they are used as target parameters of a spring in the validation process. The diagram of the proposed procedure is presented in Figure 6.

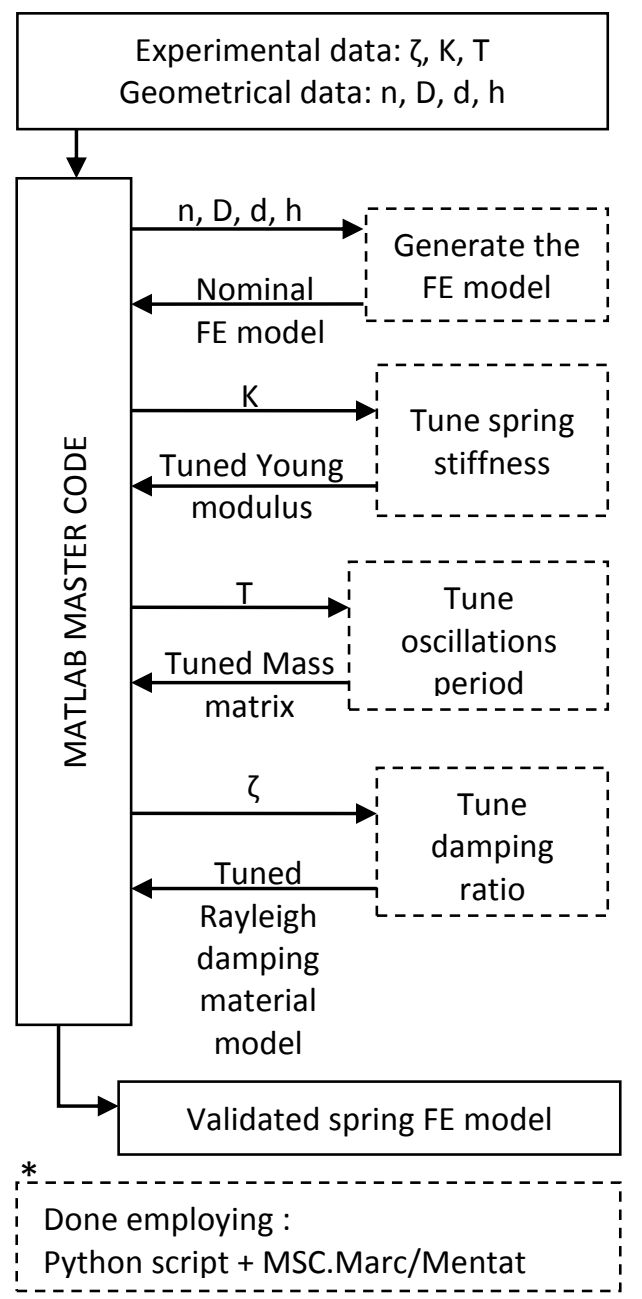

Fig. 6. Algorithm for numerical model validation.

Input data regarding the geometrical properties of the spring (n, D, d, h - spring pitch) as well as target data $(\mathrm{K}, \zeta$ and $\mathrm{T}$ - oscillations period) are passed as the input data. The first step of the procedure involves an automatic FE model generation (employing the Python programming language and the MSC.Marc/Mentat software). The Python script automatically generates commands for FE model generation, taking into account given geometric data. The spring model consists of 1D beam elements. The number of elements per one spring coil can be defined by the user. The script allows to create models of any spring, but for validation purposes the geometrical properties have to be the same as in the tested springs. Exemplary FE model of a spring generated by the developed setup is presented in Figure 2C. Initial results post-processing was performed employing Altair HyperView programme. 
Next, when the Nominal FE model is prepared, the tuning of the spring stiffness is done. Another script defines the initial Young modulus for the spring material. Then static simulation is computed and from the registered results the spring rate $\mathrm{K}$ is computed. The Young's modulus is modified and the procedure is repeated automatically in a loop using the appropriate script until the $\mathrm{K}$ parameter computed form the $\mathrm{FE}$ model is the same as the entered target value (having regard to a permissible user-defined error). The results of Young's modulus tuning are presented in Figure 7. The value of permissible error of stiffness coefficient was set to $0.001 \%$, and, after 19 iterations, the Young's modulus was finally tuned to the value of $190708 \mathrm{MPa}$ and the corresponding $\mathrm{K}$ of the spring was $3.927506 \mathrm{~N} / \mathrm{mm}$.

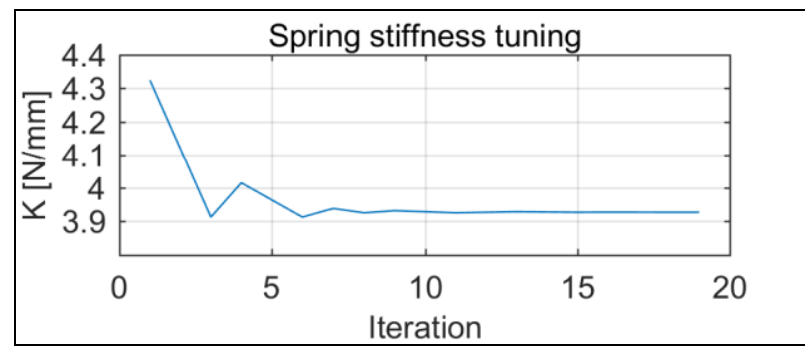

Fig. 7. Algorithm for numerical model validation.

Subsequently, the mass matrix of the FE model is tuned to get proper period of oscillations of the system (in the dynamic simulation). The mass is tuned, to finally reach the same oscillations period as in the tests. The way to reach the desired period is the same as in the spring stiffness case. The number of 9 iterations were required to tune the mass matrix with acceptable error for the oscillations period equal to $0.23 \mathrm{~s}$. It is assumed, that material damping is the only source of damping in the system. Therefore, the Rayleigh damping material model is introduced in the material of the spring. The formula describing the Rayleigh damping model is as follows:

$$
C=\sum_{i=1}^{n}\left(\alpha_{i} \cdot M_{i}+\beta_{i} \cdot K_{i}\right)
$$

where:

$\mathrm{C}$ - is the global damping matrix,

$\mathrm{M}_{\mathrm{i}}$ - is the mass matrix of $i^{\text {th }}$ element,

$\mathrm{K}_{\mathrm{i}}-$ is the stiffness matrix of $\mathrm{i}^{\text {th }}$ element,

$\alpha-$ is the mass damping multiplier,

$\beta-$ is the stiffness damping multiplier,

$\mathrm{n}-$ is the number of elements.

In case of the damping model tuning, the authors decided to investigate a finite number of combinations of the $\alpha$ and $\beta$ coefficients, because it would be much more difficult to tune automatically the parameters of damping model in contrary to the spring coefficient and mass matrix tuning. Based on the results from experimental tests, it was assumed that the damping in the system is relatively small, therefore a very low $\alpha$ and $\beta$ multipliers were assumed in the proportional damping model. In the first attempt to search the domain of $\alpha$ and $\beta$ combinations, the range of $0-0.0016$ with step of 0.0001 was chosen for both parameters. For a single combination of $\alpha$ and $\beta$ a dynamic simulation was computed and, again, employing the logarithmic decrement approach, the damping ratio of the spring was found. The results of this tuning are presented in Figure 8. In total, 256 dynamic simulations were computed to collect this data.

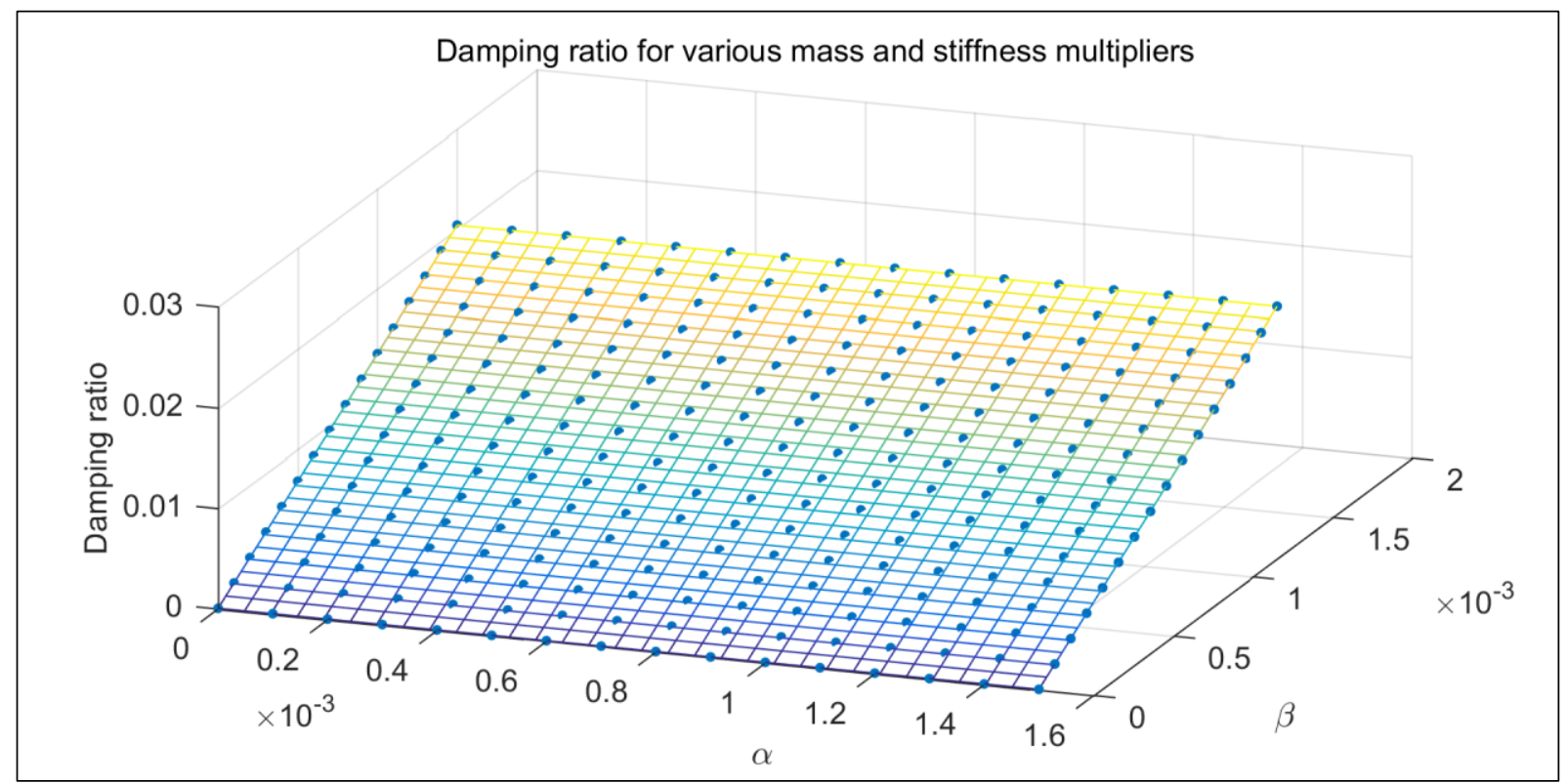

Fig. 8. Damping ratio for various $\alpha$ and $\beta$ - initial domain. 


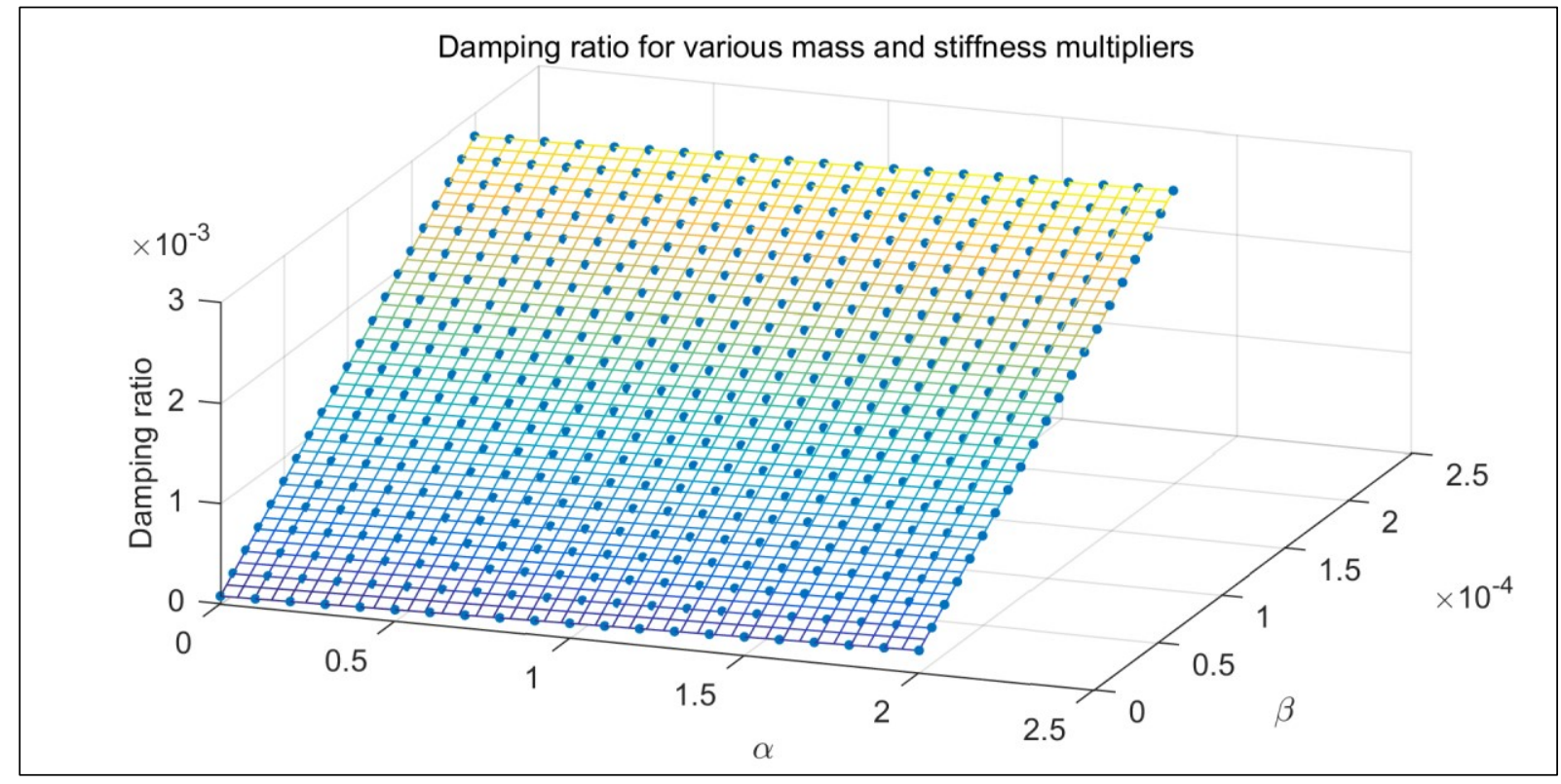

Fig. 9. Damping ratio for various $\alpha$ and $\beta$ - the second domain.

It was observed, that increasing the $\beta$ multiplier by the 0.0001 , step introduces too much damping in the system, meanwhile an increase by the same value the $\alpha$ multiplier almost does not introduce more damping in the system. Therefore the second investigation with different domain of $\alpha$ and $\beta$ parameters was done. The $\alpha$ multiplier was investigated in the range of $0-2.0$ with step of 0.1 and $\beta$ was investigated in the range of $0-2.0$ with step of 0.00001 . The results of the second domain analysis are presented in Figure 9. In total, 441 dynamic simulations were computed to collect this data. The combination of $\alpha$ and $\beta$, that corresponds to the damping ratio that is closest to the data collected in the experiment is as follows: $\alpha=0.8$ and $\beta=0.00012$. The damping ratio corresponding to these values is 0.001747 , so the relative error comparing to the experiment is $-0.17) \%$.

\section{Results of validation}

The most important achievement of the validation process was the determination of the correct material parameters. The properties of the material from which the validated spring is made are summarized in Table 2 .

Table 2. Validated material data.

\begin{tabular}{|c|c|c|c|}
\hline & Young & \multicolumn{2}{|c|}{ Rayleigh damping } \\
\cline { 3 - 4 } & modulus & $\alpha$ & $\beta$ \\
\hline Valid. material & $190708 \mathrm{MPa}$ & 0.8 & 0.00012 \\
\hline
\end{tabular}

The comparison of results (stiffness and damping ratio) experiment and from validated numerical model are presented in Table 3 .

Table 3. The results.

\begin{tabular}{|l|l|l|}
\hline & $\mathrm{K}$ & $\zeta$ \\
\hline Experiment & $3.927500 \mathrm{~N} / \mathrm{mm}$ & 0.001750 \\
\hline FE model & $3.927506 \mathrm{~N} / \mathrm{mm}$ & 0.001747 \\
\hline Relative error & $0.000153 \%$ & $-0.171428 \%$ \\
\hline
\end{tabular}

The FE numerical model was successfully validated employing the presented algorithm. The comparison (between experimental data and form the FE model) of the time histories of system oscillations are presented in Figure 10. The acceleration courses were normalized do the range $(-1,1)$,It can be observed that they are very similar to each other. They are characterized by the same oscillations period, and almost identical damping ratio (see Table 3).

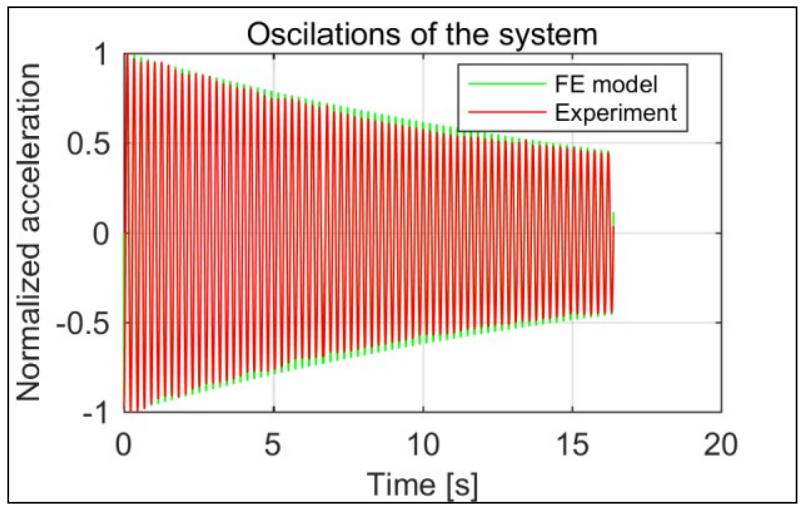

Fig. 10. Time histories of system oscillation.

\section{Modified springs analysis}

Thanks to the successful validation of the FE model, the confidence about the obtained results correctness was obtained. Therefore, other springs were investigated using the same approach and the same material data. Employing the Python script for automatic FE model generation, four models of springs with various geometrical properties were build. Subsequently, static and dynamic simulations were computed to designate $\mathrm{K}$ and $\zeta$ coefficients for the proposed alternative springs for the 160ECT pantograph collector head. Table 4 presents the results of $\mathrm{K}$ and $\zeta$ for the proposed alternative springs. 
Table 4. The results.

\begin{tabular}{|l|l|l|l|l|l|l|}
\hline & $\# 1$ & $\# 2$ & $\# 3$ & $\# 4$ & $\# 5$ & $\# 6$ \\
\hline $\mathrm{n}$ & 14.0 & 14.0 & 14.0 & 14.0 & 12.0 & 16.0 \\
\hline $\begin{array}{l}\mathrm{D} \\
{[\mathrm{mm}]}\end{array}$ & 28.0 & 24.0 & 24.0 & 20.0 & 24.0 & 24.0 \\
\hline $\begin{array}{l}\mathrm{d} \\
{[\mathrm{mm}]}\end{array}$ & 3.0 & 3.5 & 2.5 & 3.0 & 3.0 & 3.0 \\
\hline $\begin{array}{l}\mathrm{h} \\
{[\mathrm{mm}]}\end{array}$ & 6.2 & 6.2 & 6.2 & 6.2 & 6.2 & 6.2 \\
\hline $\begin{array}{l}\mathrm{K} \\
{[\mathrm{N} / \mathrm{mm}]}\end{array}$ & 2.475 & 7.273 & 1.896 & 6.780 & 4.584 & 3.435 \\
\hline$\zeta$ & 0.00142 & 0.00246 & 0.00123 & 0.00238 & 0.00194 & 0.00169 \\
\hline
\end{tabular}

These values can be used in the analysis of dynamic interaction between a pantograph and catenary and to assess if using alternative springs can be helpful in improving the quality of the pantograph - catenary cooperation. The number of new alternative springs can be easily increased in further investigations thanks to the developed computational setup.

\section{Conclusions}

The following final remarks conclude the paper:

1. Experimental research was carried out on the pantograph collector head springs. The stiffness $(\mathrm{K})$ and damping ratio $(\zeta)$ were proposed from the tests.

2. The above parameters were used in the validation process of the numerical model of the analogous springs modelled employing the FEM model.

3. The validation procedure presented in the paper allowed for successful model validation. The relative error between experimental and numerical approach is low: less than $0.001 \%$ for $\mathrm{K}$ and less than $-0.2 \%$ for $\zeta$.

4. Validated numerical model of material was used for the analysis of new collector head springs with modified geometrical parameters. The $\mathrm{K}$ and $\zeta$ were designated for alternative springs and these values can be used to investigate their influence on the pantograph - catenary interaction in further investigation.
The work was supported by the AGH Grant no. 15.11.130.627 and carried out employing the infrastructure of the Centre of Energy AGH (Czarnowiejska 36, 30-054 Krakow, Poland). The authors would like to thank the EC Engineering company for providing them with the collector head springs.

\section{References}

1. P. Zdziebko, A. Martowicz, T. Uhl, ITM Web Conf. 15, 03001 (2017)

2. P. Zdziebko, A. Martowicz, T. Uhl, in: Dynamical Systems in Applications,(Springer, 2018) (to be published)

3. P. Zdziebko, T. Uhl, Archive of Transport 39, 77-85 (2016)

4. J. Ambrósio, J. Pombo, P. Antunes, M. Pereira, Vehicle Syst. Dyn. 53, 314-328 (2015).

5. S. Bruni, J. Ambrosio, A. Carnicero, Y.H. Cho, L. Finner, M. Ikeda, S.Y. Kwon, J.P. Massat, S. Stichel, M. Tur, W. Zhang, Vehicle Syst. Dyn. 53, 412-435 (2015)

6. P.A. Jönsson, S. Stichel, C. Nilsson, Vehicle Syst. Dyn. 53, 341-346 (2015)

7. M. Tur, L. Baeza, F.J. Fuenmayor, E. García, Vehicle Syst. Dyn. 53, 402-411 (2015)

8. E. Chater, D. Ghani, F. Giri, A. Rachid, F.Z. Chaoui, M. Haloua, IFAC Proc. Vol. 46, 131136 (2013)

9. C. Sanchez-Rebollo, J.R. Jimenez-Octavio, A. Carnicero, Vehicle Syst. Dyn. 51, 554-569 (2013)

10. Y. Song, H. Ouyang, Z. Liu, G. Mei, H. Wang, X. Lu, Mech. Mach.Theory. 115, 35-59 (2017)

11. J. Pombo, J. Ambrósio, Comput. Struct. 110-111, 32-42 (2012)

12. N. Zhou, W. Zhang, Finite Elem . Anal. Des. 47, 288-295 (2011)

13. J. Ambrósio, J. Pombo, M. Pereira, TAML 3, 013006 (2013) 